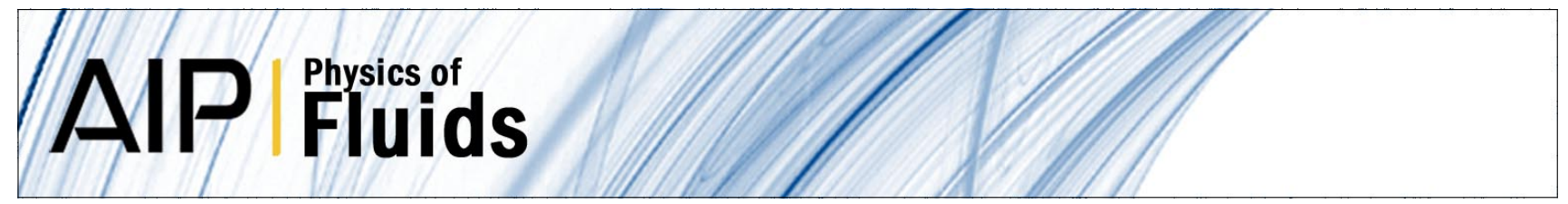

\title{
Stokes flow paths separation and recirculation cells in X-junctions of varying angle
}

M. Cachile, L. Talon, J. M. Gomba, J. P. Hulin, and H. Auradou

Citation: Phys. Fluids 24, 021704 (2012); doi: 10.1063/1.3690100

View online: http://dx.doi.org/10.1063/1.3690100

View Table of Contents: http://pof.aip.org/resource/1/PHFLE6/v24/i2

Published by the American Institute of Physics.

\section{Related Articles}

Stereoscopic high-speed imaging using additive colors

Rev. Sci. Instrum. 83, 043701 (2012)

All-optical monitoring of acoustic waves guided by confined micro-fluidic layers

Appl. Phys. Lett. 100, 144102 (2012)

Note: Development of a compact x-ray particle image velocimetry for measuring opaque flows. II. Threedimensional velocity field reconstruction

Rev. Sci. Instrum. 83, 046102 (2012)

Hydrodynamic cavitation in microsystems. II. Simulations and optical observations

Phys. Fluids 24, 047101 (2012)

Flow visualization of a vortex ring interaction with porous surfaces

Phys. Fluids 24, 037103 (2012)

\section{Additional information on Phys. Fluids}

Journal Homepage: http://pof.aip.org/

Journal Information: http://pof.aip.org/about/about_the_journal

Top downloads: http://pof.aip.org/features/most_downloaded

Information for Authors: http://pof.aip.org/authors

\section{ADVERTISEMENT}

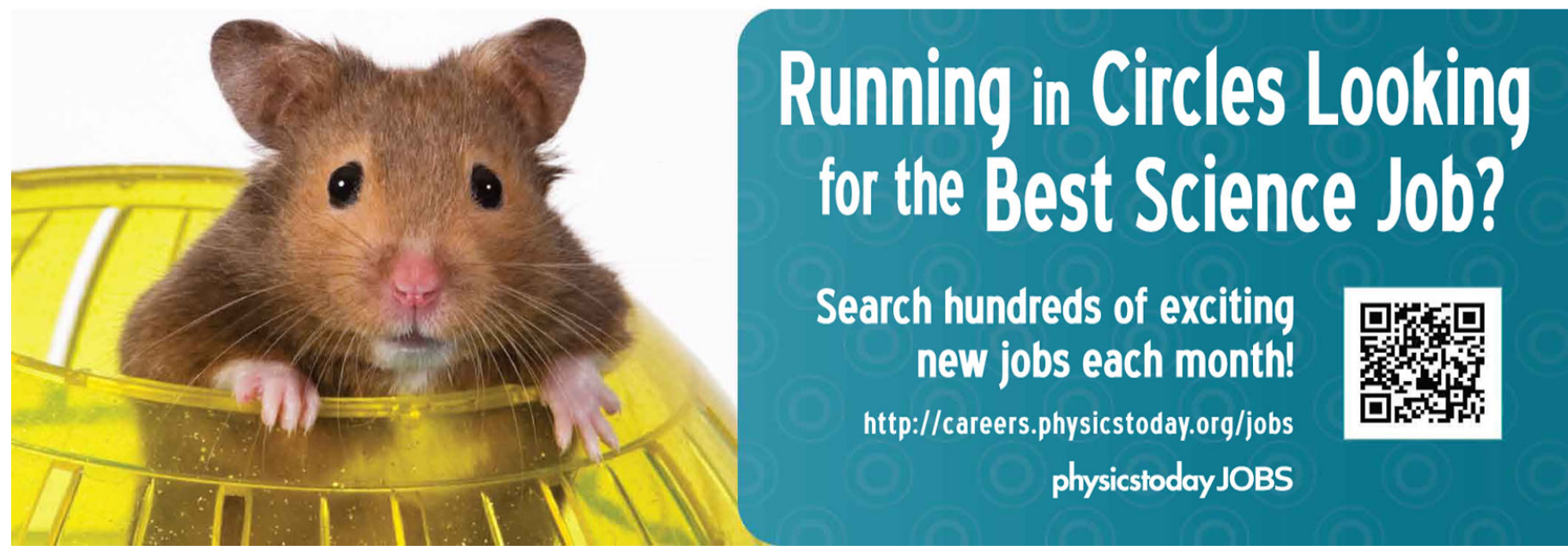




\title{
Stokes flow paths separation and recirculation cells in $\mathrm{X}$-junctions of varying angle
}

\author{
M. Cachile, ${ }^{1, a)}$ L. Talon, ${ }^{2, b)}$ J. M. Gomba, ${ }^{3, c)}$ \\ J. P. Hulin, ${ }^{2, d)}$ and H. Auradou ${ }^{2, e)}$ \\ ${ }^{1}$ Grupo de Medios Porosos, Facultad de Ingeniería, Paseo Colon 850, \\ 1063 Buenos Aires, Argentina \\ ${ }^{2}$ Univ Pierre et Marie Curie-Paris 6, Univ Paris-Sud, CNRS, F-91405. Lab FAST, \\ Bât 502, Campus Univ, F-91405 Orsay, France \\ ${ }^{3} I F A S$, UNCPBA, Tandil, Argentina
}

(Received 20 December 2011; accepted 10 February 2012;

published online 28 February 2012)

\begin{abstract}
Fluid and solute transfer in X-junctions between straight channels is shown to depend critically on the junction angle $\alpha$ in the Stokes flow regime. Experimentally, water and a water-dye solution are injected at equal flow rates in two facing channels of the junction. Planar laser induced fluorescence (PLIF) measurements show that the largest part of each injected fluid "bounces back" preferentially into the outlet channel at the lowest angle to the injection; this is opposite to the inertial case and requires a high curvature of the corresponding streamlines. The proportion of this fluid in the other channel decreases from $50 \%$ at $\alpha=90^{\circ}$ to $0 \%$ at a threshold angle. These counterintuitive features reflect the minimization of energy dissipation for Stokes flows. Finite elements numerical simulations of a 2D Stokes flow of equivalent geometry confirm these results and show that, below the threshold angle $\alpha_{c}=33.8^{\circ}$, recirculation cells are present in the center part of the junction and separate the two injected flows of the two solutions. Reducing further $\alpha$ leads to the appearance of new recirculation cells with lower flow velocities. (C) 2012 American Institute of Physics. [http://dx.doi.org/10.1063/1.3690100]
\end{abstract}

Flow control in microchannels has become an important area of research in microprocess engineering. ${ }^{1}$ The behavior of the fluids at junctions in microfluidic circuits is particularly critical for applications such as mixing, chemical reactions or heat exchange: this is for instance the case when two different fluids are injected separately into a junction of flow channels. While, for T-junctions, the flow of the two fluids at the outlet depends weakly on the junction angle at low Reynolds numbers, ${ }^{2,3}$ there are very diverse patterns in X-junctions. Then the distribution of the injected fluids between the two outlets and their interaction within the junction, which are both crucial for applications, depend strongly on the junction angle $\alpha\left(0<\alpha \leq 90^{\circ}\right)$.

For this reason, while most previous studies dealt only with orthogonal ${ }^{4}$ or parallel ${ }^{5,6}$ channels, the present work analyzes specifically the influence of the angle $\alpha$. More precisely, the two fluids are injected in facing channels and we study as a function of $\alpha$ their relative fraction in each of the outlet channels and the appearance of viscous eddies. Other authors used tangent channels at a varying angle ${ }^{7}$ but the interaction between the streams differs strongly from the present case of channels in a same plane. This system is studied by combining experimental PLIF measurements on transparent models and 2D numerical simulations. Like for Fan and Hassan, ${ }^{8}$ the experimental system is upscaled in order to visualize conveniently the flow; however, the Reynolds number

\footnotetext{
a)Electronic mail: mcachil@ fi.uba.ar.

b)Electronic mail: talon@fast.u-psud.fr.

c) Electronic mail: jgomba@exa.unicen.edu.ar.

d)Electronic mail: hulin@fast.u-psud.fr.

e)Electronic mail: auradou@fast.u-psud.fr.
} 


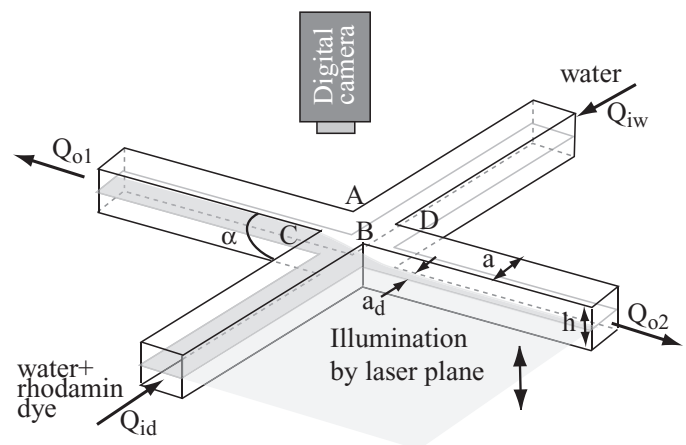

FIG. 1. Experimental setup.

$R e=\rho a U / \eta$ is low enough so that inertial effects are negligible and the Stokes equation is satisfied ( $U, \rho$, and $\eta$ are respectively the mean velocity, density, and viscosity of the fluid and $a$ is the width of the channels). The flow velocity field is therefore the same as in much smaller microchannels of same geometry.

The channels are carved into a transparent plexiglas plate by a computer controlled milling machine; both their width $a$ and depth $h$ are constant and equal to $3.55 \mathrm{~mm}$. The horizontality of the plate is carefully adjusted. Each outlet is connected to one of the inlets of a double syringe pump sucking the fluids at equal flow rates $Q_{o 1}$ and $Q_{o 2}$ (Fig. 1). The inlet channels $i w$ and $i d$ are connected to two glass flasks containing, respectively, pure water and a solution of water and rhodamin $6 \mathrm{G}$ dye $\left(0.25 \mathrm{mg} \mathrm{l}^{-1}\right)$. Due to this low concentration, the two fluids are Newtonian: their viscosities and densities are so close when their temperatures are matched that no buoyancy or viscosity driven instabilities may appear. Each bottle is placed on computer controlled scales allowing for a measurement of the flow rates $Q_{i w}$ and $Q_{i d}$ in the corresponding outlet. In the present work, these flow rates are set to be equal within $\pm 0.3 \%$ by adjusting the relative levels of the fluid in the flasks.

The distribution of the two fluids in the intersection is visualized by the PLIF technique. A plane horizontal green laser sheet of wavelength $532 \mathrm{~nm}$ and thickness $\simeq 1 \mathrm{~mm}$, parallel to both channels, enters the plexiglas model from the side. The parts of the channels containing the rhodamin dye solution are easily identified by the emission of a yellow fluorescence light of intensity proportional to the local dye concentration. The distribution of this solution in the illuminated plane is then recorded by a digital camera located above the model: unwanted reflected light from the laser is eliminated by a narrow bandwidth notch filter corresponding to its wave length $\lambda=532 \pm 25 \mathrm{~nm}$ and placed in front of the camera sensor. Regions in which only water is present appear as dark while those containing rhodamin are illuminated (Fig. 2). In the present work, our analysis is based on the geometry of the boundary between the zones occupied by the two fluids. The laser is mounted on a vertical translation stage: this allowed us to scan the distribution of the fluids at different heights $z$ for several Reynolds numbers $(0.5 \leq R e \leq 50)$.

The distributions of the fluids in the symmetry plane at different $R e$ values have first been compared in order to check the validity of the linear Stokes equation. For $R e=0.5$ and $R e$ $=5$, the geometry of the boundary between the transparent and dyed fluid is the same: this implies that the linearity condition is satisfied and that the flow is purely viscous. At higher $R e$ values $(R e \gtrsim 15)$, inertial effects induce, as expected, changes of the shape of the boundary. Moreover, for $R e=0.5$ and $R e=5$, there is no visible influence of the vertical coordinate $z$ of the light sheet: the distribution of the two fluids can then be considered as bidimensional. The flow field itself is, however, not bidimensional because of the vertical velocity gradients created by the upper and lower wall: however, the orientation of the velocity and, therefore, the streamlines are approximately invariant with $z$. In contrast, for $R e \gtrsim 40,3 \mathrm{D}$ structures appear in the fluid distribution. The boundary between the dyed and the transparent fluid in the images is fuzzier for $R e=0.5$ than for $R e=5$ and the thickness of the transition zone increases with distance to a value of the order of $0.5 \mathrm{~mm}$ for a path length of the order of a $\mathrm{cm}$. This value is of the same order of magnitude as that corresponding to transverse molecular diffusion (using $D_{m}=2.8 \times 10^{-10} \mathrm{~m}^{2} \mathrm{~s}^{-1}$ at $T=22{ }^{\circ} \mathrm{C}$ (Ref. 9)). 

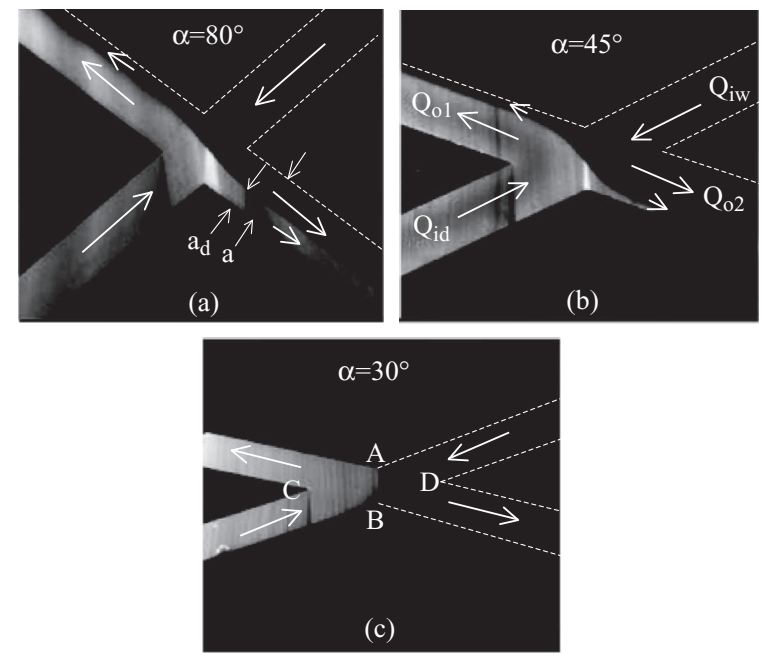

FIG. 2. Experimental images displaying the distribution of the fluids in the junction for $R e=5$. (a) $\alpha=80^{\circ}$, (b) $\alpha=45^{\circ}$, (c) $\alpha=30^{\circ}$.

Taking into account the above results, all experiments reported below were performed at $R e=5$ for which both sharp boundaries and Stokes flow are obtained. Cells with 6 different junctions angles $\alpha=90^{\circ}, 80^{\circ}, 45^{\circ}, 30^{\circ}, 20^{\circ}$, and $10^{\circ}$ have been used. Figure 2 displays pictures obtained in the stationary regime for three angles $\alpha=80^{\circ}, 45^{\circ}$, and $30^{\circ}$. The dark and illuminated regions of the junction are symmetrical with respect to the center of symmetry of the junction: this confirms that the flow rates $Q_{i d}$ and $Q_{i w}$ at the two inlets are equal. A first key observation is that, for all angles $\alpha$, each injected fluid flows dominantly towards the outlet at the lowest angle with the injection channel (outlet 1 for the fluorescent solution): this corresponds to flow paths with a higher curvature than those leading to the other outlet. The flow of each fluid is equally split between the outlets only for $\alpha=90^{\circ}$. At $\alpha=45^{\circ}$, the fraction of dyed fluid moving towards outlet 2 is small and is exactly zero for $\alpha=30^{\circ}$. In this latter case, all incoming streamlines "bounce back" on the junction towards outlet 1 . The main flows of the two fluids are then completely separated.

More quantitatively, the distribution of each fluid between the two outlets will be characterized by the volume fraction $q$ occupied by the fluid of interest: in practice, $q$ is taken to be equal to $a_{d} / a$ in which $a$ is the total width of the section and $a_{d}$ the width of the flow tube corresponding to this fluid in the parallel flow region. Here, $a_{d}$ is computed for the dyed solution in outlet 2 where its volume fraction is lower (Figs. 1 and 2(a)). Several measurements of $a_{d}$ in the parallel flow region are averaged in order to compute $q$. Figure 3 displays the experimental variation (• symbols) of the fraction $q$ with $\alpha: q$ is exactly zero below a threshold angle $\alpha_{c}$ of experimental value in the range $30^{\circ} \leq \alpha_{c} \leq 35^{\circ}$. Above $\alpha_{c}, q$ increases linearly with $\alpha$ up to $q=0.5$ for $\alpha=90^{\circ}$.

The zero value of $q$ below the critical angle $\alpha_{c}$ may seem at first counter-intuitive: it implies indeed that the fluid particles follow preferentially, in the region of the junction, the streamlines with the highest curvature. Actually, this reflects a key characteristic of all viscous Stokes flows, namely that they must achieve a minimum viscous energy dissipation. Let us assume that, in contrast to the experiment of Fig. 2(c), the dyed fluid flows into outlet 2 and the transparent one into 1 at low values of $\alpha$. This requires a counterflow of the two fluids along segment $C D$ which is much longer than $A B$; moreover, this small length of $A B$ increases the transverse velocity gradient. The viscous energy dissipation, proportional to the integral of the square of this gradient, would then be too high. In the experimental flow of Fig. 2(c), instead, the distance $A B$ along which the two flows coexist is smaller than the transverse distance $C D$ : together with the appearance of recirculation cells with very low velocities (and gradients), this leads, indeed, to a lower dissipation than in the first case. The result would be completely different for the inertial potential flow of a perfect fluid in the same geometry. Let us consider, for instance, the comparable classical problem of a $2 \mathrm{D}$ jet of perfect fluid 


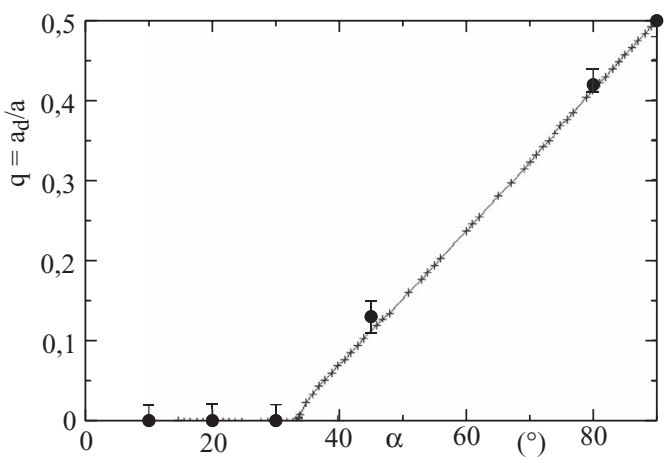

FIG. 3. Variation of ratio $q=a_{d} / a$ (see Fig. 2(a)) as a function of the junction angle $\alpha$. (•) experimental measurements; (+) results of the numerical simulations.

impinging obliquely at an angle $\alpha$ on a plane wall: ${ }^{10}$ then, as $\alpha$ decreases from $90^{\circ}$ to $0^{\circ}$, the fraction (equivalent to $q$ ) of the fluid following the path with the lowest curvature increases from 0.5 to 1 (instead of decreasing to 0 like in Fig. 3).

The structure of the flow in the center part of the junction is obviously a key element for predicting the exchanges between the two flowing fluids. Additional information is obtained by saturating the intersection with the rhodamin-water solution instead of pure water before establishing a stationary flow in the facing injection channels. No difference is observed for $\alpha>\alpha_{c}$ but, for $\alpha<\alpha_{c}$, the fluorescence light intensity is higher in the center of the junction than when it was initially saturated by transparent fluid. This suggests that some dye is trapped in the center of the junction and that the latter corresponds to a dead or recirculation zone.

In order to verify this assumption, the $2 \mathrm{D}$ Stokes equation has been solved numerically in the $(x, y)$ plane by means of the FreeFem finite elements package: ${ }^{11}$ the lack of inertial effects and the 2D distribution of the two fluids suggest indeed that this equation may predict adequately the distribution of the two fluids. The velocity vector field and the sample streamlines obtained in this way for three values of the angle $\alpha$ are displayed in Fig. 4. For $\alpha=31^{\circ}$ (Fig. 4(c)), a recirculation cell is observed in the center of the junction as suggested by the experiments. It separates completely the flows on the two sides of the junction. The boundary of this zone inside which the flow lines are not connected to the outside is marked by dashed lines. The modulus of the velocity (not represented in the figure) is about 100 times lower inside the cell than outside. The detailed structure of the recirculation flow depends on the angle $\alpha$ and will be discussed below. For $\alpha=40^{\circ}$ (Fig. 4(a)), there is only one stagnation point and it is located at the center of symmetry of the junction: streamlines originating at this point separate those extending across the junction from those which "bounce back" on it. The distance of these particular streamlines to the nearest wall represents therefore the distance $a_{d}$ defined above. This provides a numerical prediction of the ratio $q=a_{d} / a$ : its variation as a function of the angle $\alpha$ is displayed by (+) symbols in Fig. 3. The numerical values of $q$ are first very close to the experimental ones: this supports (a posteriori) the 2D approximation. The variation of $q$ with $\alpha$ is observed to be very linear except close to the critical angle $\alpha_{c}$ : this confirms the trend of the experimental data. These simulations provide the precise value $\alpha_{c}=33.8 \pm 0.2^{\circ}$. For $\alpha=\alpha_{c}$ (Fig. 4(b)), one observes two recirculation cells on the lateral edges extending up to the central stagnation point. For this and lower angles, there is no flow path connecting the left and right sides of the junction any longer.

The recirculation zones observed at low angles $\alpha$ clearly play a major part in the exchange of passive scalars (solutes, heat, etc.) between the injected flows. Their structures depend strongly on $\alpha$ as shown in Figure 5 displaying typical examples obtained numerically. For $\alpha$ slightly above $\alpha_{c}$ (Fig. 5(a)), side recirculation cells are present, like for $\alpha=\alpha_{c}$ : they reduce the section available in the plane of symmetry (vertical in the figure) for streamlines crossing from one side to the other but do not block it. This section becomes zero for $\alpha=\alpha_{c}$ as the two cells touch each other (Fig. 4(b)) and then, below $\alpha_{c}$, a single cell extending all across the junction appears (Fig. 4(c)). The faster variation of $q$ near $\alpha_{c}$ in Fig. 3 may reflect the scarcity of the flow paths connecting the two sides 


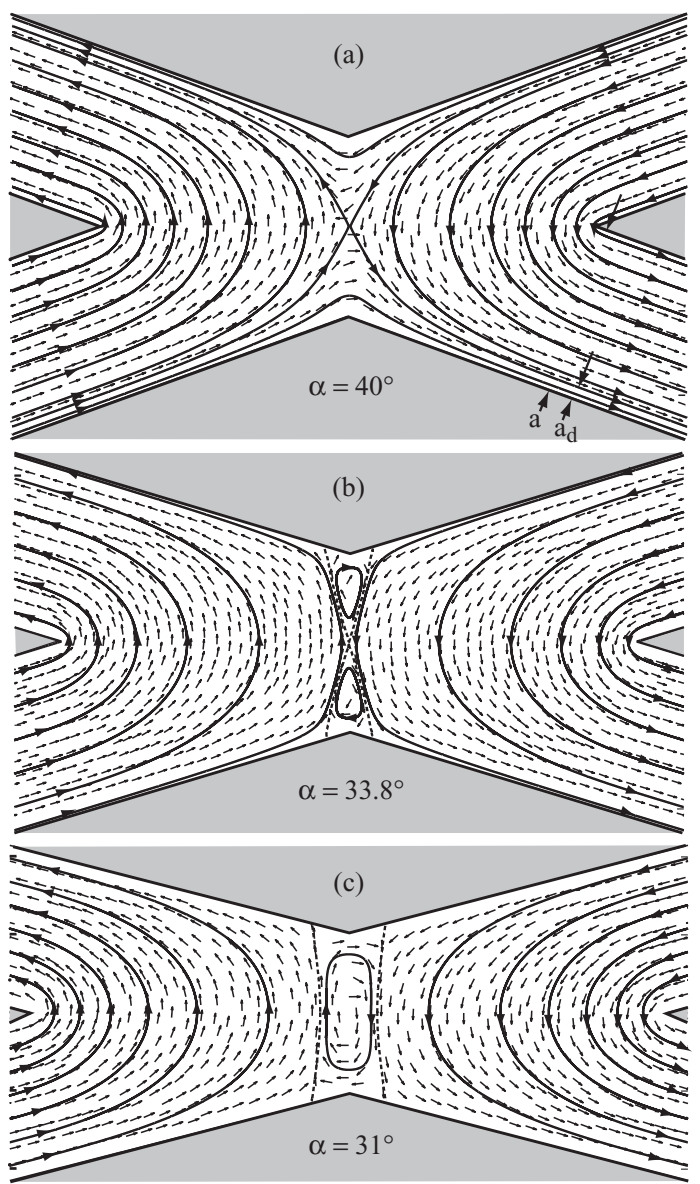

FIG. 4. Velocity field (vectors) obtained numerically for: (a) $\alpha=40^{\circ}$; (b) $\alpha=\alpha_{c}=33.8^{\circ}$; (c) $\alpha=31^{\circ}$. The length of all vectors are equal for a better visibility so that only the orientation of the velocity is represented. Continuous lines represent selected streamlines of the flow; dashed lines mark the boundaries of the flow recirculation regions.

of the junction and the fact that they cross low velocity regions. Decreasing further $\alpha$ (Fig. 5(b)) results in an elongation of the cell in the $x$ direction with, inside it, two smaller cells joined at a stagnation point (Fig. 5(b)). At a still lower angle, the latter cells occupy nearly the full width of the channel (Fig. 5(c)) until an additional one, elongated in the $y$ direction, appears in the center (Fig. 5(d)). The typical velocity decreases by a large factor $(\simeq 100)$ between the outer flow and the first gener-

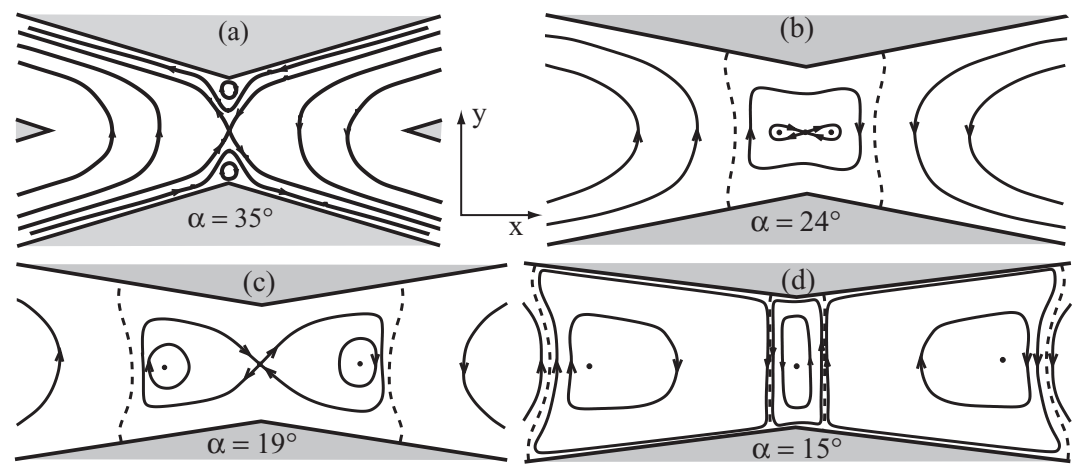

FIG. 5. Typical streamlines obtained numerically for different angles (a) $\alpha=35^{\circ}$, (b) $\alpha=24^{\circ}$, (c) $\alpha=19^{\circ}$, (d) $\alpha=15^{\circ}$. Continuous and dashed lines have the same meaning as in Fig. 4. 
ation of cells and between the latter and the next generation. New cells appear at still lower angles (not shown in the figure) and correspond to vanishingly small velocities.

Vortices have already been observed in Stokes flow by many other authors. A classical example is Moffatt vortices induced by an outside flow in a corner of angle $\beta<146^{\circ}$ between plane walls: an infinite sequence of eddies appears with velocities decreasing strongly, like here, from one generation to the next. ${ }^{12,13}$ Here, a similar sequence appears at low angles $\alpha$ but is interrupted at the middle of the junction with a symmetrical sequence starting on the other side. These Stokes flows in corners do not display, however, like in Figs. 5(a)-5(c) pairs of corotating vortices separated by a stagnation point unless a planar Couette flow is superimposed. ${ }^{14}$ These latter structures are also observed in elongated rectangular cavities when the two smaller parallel walls are sheared in opposite directions. ${ }^{15}$ Like at low $\alpha$ values in the present system, as the elongation of the cavity increases, new recirculation cells appear: one observes first a pair of vortices and they coalesce into a single cell when the elongation is increased further.

The experiments and simulations presented here demonstrate that, at all $\alpha$ values (except $90^{\circ}$ ), the largest part of (if not all) each injected flow bounces back into the outlet at the angle $\alpha$ to the injection. The actual fraction depends strongly on $\alpha$, as shown above, but will also vary with the aspect ratio $h / a$ of the section: for instance, if $h / a \ll 1$ (instead of $h / a=1$ ), one obtains a Hele Shaw cell geometry. Then the average of the velocity field over the depth $h$ would be similar to that for a perfect fluid and the variation of $q$ with $\alpha$ will be completely different. It will be important to study the transition between these two opposite behaviors by varying continuously $h / a$. Since the flow field is controlled by the viscous dissipation, it is likely to be strongly influenced by the rheological properties of the fluid. Moreover, anomalies are known to occur for fluids of high extensional viscosity in the vicinity of the stagnation points in different flow geometries. ${ }^{16-19}$ For viscoelastic fluids flowing in orthogonal channels, elastic instabilities have indeed been observed experimentall $y^{20}$ and flow asymmetries have been predicted numerically. ${ }^{21}$

The recirculation cells appearing at low $\alpha$ values will control the exchange of solute (or other passive or reactive species) between the two injected fluids: this exchange involves a combination of transverse molecular diffusion between the cells and the two flows (and from one cell to another) and convective transport by the circulation within the cells. For reactive fluids, the recirculation cells may act as microreactors into which species contained in the injected fluids are transferred through transverse molecular diffusion. Finally, it may be possible to induce a more efficient chaotic mixing in the recirculation cell by varying the geometry of the intersection and/or by using suitable time variations of the relative values of the two injection flow rates.

We thank R. Pidoux, L. Auffay, and A. Aubertin for realizing and developing the experimental set up, D. Etien for his contribution to the experiments and D. Salin, E. J. Hinch, and H. A. Stone for illuminating suggestions. We acknowledge the support of the RTRA "Triangle de la Physique" and of the LIA PMF-FMF (Franco-Argentinian International Associated Laboratory in the Physics and Mechanics of Fluids).

${ }^{1}$ N. T. Nguyen and Z. Wu, "Micromixers—a review," J. Micromech. Microeng. 15, R1 (2005).

${ }^{2}$ S. Thomas and T. A. Ameel, "An experimental investigation of moderate Reynolds number flow in a T-channel," Exp. Fluids 49, 1231 (2010).

${ }^{3}$ S. Thomas, T. A. Ameel, and J. Guilkey, "Mixing kinematics of moderate Reynolds number flows in a T-channel," Phys. Fluids 22, 013601 (2010).

${ }^{4}$ M. Hellou and T. D. P. Bach, "Stokes flow in a junction of two-dimensional orthogonal channels," Z. Angew. Math. Phys. 62, 135 (2011).

${ }^{5}$ T. Cochrane, K. Walters, and M. F. Webster,"On Newtonian and non-Newtonian flow in complex geometries,” Philos. Trans. R. Soc. London, Ser. A 301, 163 (1981).

${ }^{6}$ J.-T. Jeong, "Slow viscous flow in a partitioned channel," Phys. Fluids 13, 1577 (2001).

${ }^{7}$ D. Lee, Y.-T. Chen, and T.-Y. Bai, “A study of flows in tangentially crossing micro-channels," Microfluid. Nanofluid. 7, 169 (2009).

${ }^{8}$ Y. F. Fan and I. Hassan, "Experimental and numerical investigation of a scaled-up passive micromixer using fluorescence technique," Exp. Fluids 49, 733 (2010).

${ }^{9}$ P.-O. Gendron, F. Avaltroni, and K. J. Wilkinson, "Diffusion coefficients of several rhodamine derivatives as determined by pulsed field gradient-nuclear magnetic resonance and fluorescence correlation spectroscopy," J. Fluoresc. 18, 1093 (2008).

${ }^{10}$ E. Guyon, J. Hulin, L. Petit, and C. Mitescu, Physical Hydrodynamics (Oxford University Press, Oxford, 2001), Chap. 5, Sec. 5.4.1. 
${ }^{11}$ O. Hecht, O. Pironneau, A. le Hyaric, and K. Ohtsuka, "FreeFem++", Laboratoire Jacques-Louis Lions, Université Pierre et Marie Curie, Paris; see http://www.freefem.org/ff++/.

${ }^{12}$ H. K. Moffatt, "Viscous and resistive eddies near a sharp corner," J. Fluid Mech. 18, 1 (1964).

${ }^{13}$ J. Jeffrey and J. D. Sherwood, "Streamline patterns and eddies in low-Reynolds-number flow," J. Fluid Mech. 96, 315 (1980).

${ }^{14}$ M. C. T. Wilson, P. H. Gaskell, and M. D. Savage, "Nested separatrices in simple shear flows: The effect of localized disturbances on stagnation lines," Phys. Fluids 17, 093601 (2005).

${ }^{15}$ F. Gürcan, P. H. Gaskell, M. D. Savage, and M. C. T. Wilson, "Eddy genesis and transformation of Stokes flow in a double-lid-driven cavity,” Proc. Inst. Mech. Eng., Part C: J. Mech. Eng. Sci. 217, 353 (2003).

${ }^{16}$ M. G. N. Perera, A. Lyazid, and O. Scrivener, "Numerical simulation of elongational flow behaviour in the cross cell experiments," Rheol. Acta 21, 543 (1982).

${ }^{17}$ A. Chow, A. Keller, A. J. Müller, and J. A. Odell, "Entanglements in polymer solutions under elongational flow: A combined study of chain stretching, flow velocimetry, and elongational viscosity," Macromolecules 21, 250 (1988).

${ }^{18}$ O. G. Harlen, J. M. Rallison, and M. D. Chilcott, "High-Deborah-number flows of dilute polymer solutions," J. Non-Newtonian Fluid Mech. 34, 319 (1990).

${ }^{19}$ O. J. Harris and J. M. Rallison, "Start-up of a strongly extensional flow of a dilute polymer solution," J. Non-Newtonian Fluid Mech. 50, 89 (1993).

${ }^{20}$ P. E. Arratia, C. C. Thomas, J. Diorio, and J. P. Gollub, "Elastic instabilities of polymeric solutions in cross-channel flow," Phys. Rev. Lett. 96, 144502 (2006).

${ }^{21}$ R. J. Poole, M. A. Alves, and P. J. Oliveira, "Purely elastic flow asymmetries," Phys. Rev. Lett. 99, 164503 (2007). 